\title{
A Fourier-Cosine Method for Pricing Discretely Monitored Barrier Options under Stochastic Volatility and Double Exponential Jump
}

\author{
Shoude Huang $(\mathbb{D}$ and Xunxiang Guo \\ School of Economic Mathematics, Southwestern University of Finance and Economics, Chengdu 611130, Si Chuan, China \\ Correspondence should be addressed to Xunxiang Guo; xguo3@swufe.edu.cn
}

Received 23 May 2020; Accepted 30 September 2020; Published 13 October 2020

Academic Editor: Wenguang Yu

Copyright (c) 2020 Shoude Huang and Xunxiang Guo. This is an open access article distributed under the Creative Commons Attribution License, which permits unrestricted use, distribution, and reproduction in any medium, provided the original work is properly cited.

In this paper, the valuation of the discrete barrier options on the condition that the underlying asset price process follows the GARCH volatility and double exponential jump is studied. We derived an analytical approximation of the characteristic function for the underlying log-asset price. Then, a quasianalytical approximate formula of the price of the discrete barrier option is obtained based the on Fourier-cosine method. Numerical examples show that the Fourier-cosine method is fast and efficient for pricing discrete barrier options compared with the Monte Carlo simulation method. Finally, the influences of some important parameters on the prices of discrete barrier options are studied to further illustrate the rationality of the model.

\section{Introduction}

Since it was proposed in 1973, the well-known BlackScholes-Merton (BS) model [1] is often applied in financial industries because the analytic solution of European option under the risk-neutral measure has been obtained by assuming that the underlying price process follows the geometric Brownian motion. In order to hedge and manage the risk, more options were created, such as American options and exotic options. Barrier option is one of the most actively traded exotic options in international financial derivative markets because of lower cost. For example, the gross market value of the Over-the-Counter (OTC) barrier options accounted for a significant proportion in the US foreign exchange option market in 2018. And many participants maintain considerable barrier option portfolios, which call for associated valuation and risk management tools for these securities in foreign exchange markets.

In view of the important role of barrier options, barrier option pricing is a significant problem in the theoretical researches and applications. Under the BS model framework, closed-form solutions for all kinds of European style barrier options have been obtained [2-4]. But many studies have shown that jump exists in the prices of various financial assets, such as stocks and commodities, especially after financial crises or major natural disasters happen, while the above-mentioned literatures did not take jumps into account. Kou and Wang [5] proposed a jump-diffusion model and obtained analytical solution of the European style barrier options using the Laplace transform method, where the jump size is assumed to follow a double exponential distribution.

In financial engineering, the discretely monitored barrier options are becoming a research focus. A growing number of researchers are becoming interested in the valuation of discretely monitored options. Feng and Linetsky [6] proposed the fast Hilbert transform method to obtain the price of the discretely monitored barrier options under Lévy models. Fusai, Germano, and Marazzina [7] utilized Wiener-Hopf factorization to obtain the price of discretely monitored barrier options under exponential Lévy models. Lian, Zhu, Elliott, and Cui [8] proposed a semianalytical solution for the valuation of the discrete barrier options given that the underlying asset price process was driven by a time-dependent Lévy process. Sobhani and Milev [9] presented a Legendre multiwavelets method for pricing discrete double barrier options. Thakoor, Tangman, and Bhuruth [10] proposed a novel spectral method by discretizing the 
pricing equation of the discrete barrier options whose price process is modelled by the stochastic Alpha Beta Rho (SABR) model. Liu and Zhang [11] proposed a novel jumpdiffusion model in present of liquidity risk and derived an approximate solution for the valuation of the discrete barrier options.

Unfortunately, these mentioned models are assumed that the volatility is constant. This is different from the wellknown facts of empirical researches such as volatility smile or smirk. To overcome these shortcomings, many researchers proposed stochastic volatility models, among which the popular models are raised by Stein and Stein [12], Heston [13]. But a large number of studies have shown that the square-root process setup of the Heston model does not perform well in applications and cannot describe some of the stylized facts observed from the financial time series. For the past few years, the generalized autoregressive conditional heteroskedasticity (GARCH) model proposed by Nelson [14] has attracted extensive attention. Christoffersen P.et al. [15] and Chourdakis and Dotsis [16] further studied the GARCH model and found that the GARCH model describes the features of observations from the option markets much better than other stochastic volatility models, including the Heston model. In addition, Huang et al. [17] reached the same conclusion after their empirical study on Shanghai Stock Exchange (SSE) 50 ETF option.

In this paper, we focus on the valuation of discretely monitored barrier options under the GARCH volatility and double exponential jump. Since there is no analytical formula for the characteristic function of the underlying asset log-price, a perturbation method is applied to derive an analytical approximation of the characteristic function for the log-price of the underlying asset. Moreover, we obtain an approximate price for the discretely monitored barrier option using the Fourier-cosine method proposed by Fang and Oosterlee [18]. Fourier-cosine method, as an alternative to the fast Fourier transform, was firstly proposed by Fang and Oosterlee. It can improve the speed of pricing and achieve an exponential convergence rate for European options. This method has been widely used in option pricing, such as Fang and Oosterlee [19, 20], Zhang and Geng [21], and Li and Zhang et al. [11]. Finally, some numerical simulations and sensitivity analysis are proposed to explain our results, and some economic implications of these results are discussed to demonstrate some interesting phenomena.

An outline of this paper is as follows. Section 2 sets up an option pricing model based on the GARCH volatility and double exponential jump. The approximation of the characteristic function for the underlying asset log-asset price is derived. And an approximate price for discretely monitored barrier option is also derived in Section 3. Some numerical examples and sensitivity analysis are provided to explain our results in Section 4. We conclude the paper in Section 5 .

\section{Model Specification}

Let $\left\{\Omega, \mathscr{F},\left\{\mathscr{F}_{t}\right\}_{0 \leq t \leq T}, \mathbb{Q}\right\}$ be a complete probability space with a filtration continuous on the right line, where $\mathbb{Q}$ is a risk-neutral probability. The price process of the underlying asset $S_{t}$ and the process of the volatility $v_{t}$ are specified under the risk-neutral measure $\mathbb{Q}$ as follows:

$$
\begin{aligned}
& \frac{\mathrm{d} S_{t}}{S_{t}}=(r-\lambda m) \mathrm{d} t+\sqrt{v_{t}} \mathrm{~d} W_{s, t}+\left(e^{J}-1\right) \mathrm{d} N_{t}, \\
& \mathrm{~d} v_{t}=k\left(\theta-v_{t}\right) \mathrm{d} t+\sigma v_{t}\left(\rho \mathrm{d} W_{s, t}+\sqrt{\left(1-\rho^{2}\right)} \mathrm{d} W_{v, t}^{\perp}\right),
\end{aligned}
$$

where $\operatorname{Cov}\left(\mathrm{d} W_{s, t}, \mathrm{~d} W_{v, t}\right)=\rho \mathrm{d} t$. And $k, \theta, \sigma$ mean the speeds of the mean reversion, long-run volatility, and instantaneous volatility of the process $v_{t}$, respectively. $N_{t}$ is a Poisson process with constant intensity $\lambda, m=E^{\mathbb{Q}}\left(e^{J}-1\right)$. Jump size $J$ is a random variable and follows an asymmetric double exponential distribution with the density

$$
f(J)=p \eta_{1} e^{-\eta_{1} J} 1_{\{J \geq 0\}}+q \eta_{2} e^{\eta_{2} J} 1_{\{J<0\}}, \quad \eta_{1}>1, \eta_{2}>0,
$$

where $p, q \leq 1, p+q=1$ are probability of up-move jump and probability of down-move jump, respectively. So we can obtain that $m=p \eta_{1} / \eta_{1}-1+q \eta_{2} / \eta_{2}+1-1$.

Let $x_{t}=\ln S_{t} / K$. We can transform the equations (1) and (2) into the following equations (4) and (5), respectively:

$$
\begin{aligned}
& \mathrm{d} x_{t}=\left(r-\lambda m-\frac{v_{t}}{2}\right) \mathrm{d} t+\sqrt{v_{t}} \mathrm{~d} W_{s, t}+J \mathrm{~d} N_{t}, \\
& \mathrm{~d} v_{t}=k\left(\theta-v_{t}\right) \mathrm{d} t+\sigma v_{t}\left(\rho \mathrm{d} W_{s, t}+\sqrt{\left(1-\rho^{2}\right)} \mathrm{d} W_{v, t}^{\perp}\right) .
\end{aligned}
$$

\section{Valuation of Discretely Monitored Barrier Options}

In this section, we derived the approximate characteristic function of the underlying log-asset price. Then we obtain the approximate prices of discretely monitored barrier options via the Fourier-cosine method based on the proposed model.

\subsection{Derivation of Approximate Characteristic Function.} Following Caldana and Fusai [22], the characteristic function of $x_{T}$ under the risk-neutral $\mathbb{Q}$ is defined by

$$
\Phi(x, v, \tau ; u)=E^{\mathbb{Q}}\left[e^{i u x_{T}} \mid t x_{t} n=q x h, v_{t} x=7 v\right],
$$

where $T \geq t, \tau=T-t, i=\sqrt{-1}$. Then we can obtain the following theorem. 
Theorem 1. Given that the underlying asset price follows the dynamics in equations (1) and (2). The approximate characteristic function for $x_{T}$ is given by

$$
\Phi(x, v, \tau ; u)=\exp (i u x+A(u, \tau) v+B(u, \tau))
$$

where

$$
\begin{aligned}
& A(u, \tau)= \alpha_{0} \frac{1-e^{-\alpha \tau}}{-\beta_{2}+\beta_{1} e^{-\alpha \tau}} \\
& B(u, \tau)=-\frac{1}{2} \theta A(\tau, u) \\
&-\frac{\alpha_{3}}{\alpha_{2}}\left[\beta_{1} \tau+\ln \left(\frac{-\beta_{2}+\beta_{1} e^{-\alpha \tau}}{\alpha}\right)\right] \\
&+((r-\lambda m) i u+\lambda \Lambda(u)) \tau-\frac{1}{4} \theta\left(i u+u^{2}\right) \tau, \\
& \alpha_{0}=-\frac{1}{2}\left(i u+u^{2}\right) \\
& \alpha_{1}= \frac{3}{2} \theta^{\frac{1}{2}} \sigma \rho i u-k \\
& \alpha_{2}= \sigma^{2} \theta \\
& \beta_{1}= \frac{\alpha_{1}+\alpha}{2}, \\
& \alpha_{3}= \frac{1}{2} \theta k+\frac{1}{4} \sigma \rho i u \theta^{3 / 2} . \\
& \beta_{2}= \frac{\alpha_{1}-\alpha}{2}, \\
& \alpha_{1}^{2}-4 \alpha_{0} \alpha_{2} \\
& \hline
\end{aligned}
$$

Proof. By Feynman-Kac theorem, $\Phi(x, v, \tau ; u)$ satisfies the following PIDE:

$$
\begin{aligned}
& -\frac{\partial \Phi}{\partial \tau}+\left(r-\frac{1}{2} v-\lambda m\right) \frac{\partial \Phi}{\partial x}+\frac{1}{2} v \frac{\partial^{2} \Phi}{\partial x^{2}} \\
& +k(\theta-v) \frac{\partial \Phi}{\partial v}+\frac{1}{2} \sigma^{2} v^{2} \frac{\partial^{2} \Phi}{\partial v^{2}} \\
& +\frac{\partial^{2} \Phi}{\partial x \partial v} v^{3 / 2} \sigma \rho+\lambda \int_{-\infty}^{+\infty}[\Phi(x+J)-\Phi(x)] f(J) \mathrm{d} J=0 .
\end{aligned}
$$

The boundary condition for equation (9) is given by

$$
\Phi(x, v, 0 ; u)=e^{i u x_{T}} .
$$

Notice that the integral term in equation (9),

$$
\begin{aligned}
& \int_{-\infty}^{+\infty}[\Phi(x+J)-\Phi(x)] f(J) \mathrm{d} J \\
& =\int_{-\infty}^{+\infty}\left[E^{\mathbb{Q}}\left[e^{i u(x+J)}\right]-E^{\mathbb{Q}}\left[e^{i u x}\right]\right] f(J) \mathrm{d} J \\
& =\int_{-\infty}^{+\infty}\left[E^{\mathbb{Q}}\left[e^{i u x}\left(e^{i u J}-1\right)\right]\right] f(J) \mathrm{d} J \\
& =\int_{-\infty}^{+\infty} E^{\mathbb{Q}}\left[e^{i u x}\right] E^{\mathbb{Q}}\left[e^{i u J}-1\right] f(J) \mathrm{d} J \\
& =\Phi(x, v, \tau ; u) \Lambda(u),
\end{aligned}
$$

where $\Lambda(u)=p \eta_{1} / \eta_{1}-i u+q \eta_{2} / \eta_{2}+i u-1$. Equation (9) is very difficult to solve since it is a nonlinear PDE. So we first linearize it approximately. The idea is to approximate $v^{3 / 2}, v^{2}$ in the PIDE using Taylor expansions around the long-run mean of variance as follows:

$$
\begin{gathered}
v^{2} \approx 2 \theta v-\theta^{2}, \\
v^{3 / 2} \approx \frac{3}{2} \theta^{1 / 2} v-\frac{1}{2} \theta^{3 / 2} .
\end{gathered}
$$

Substituting equations (11)-(13) into the PDE in equation (9), we have

$$
\begin{aligned}
& -\frac{\partial \Phi}{\partial \tau}+\left(r-\frac{1}{2} v-\lambda m\right) \frac{\partial \Phi}{\partial x}+\frac{1}{2} v \frac{\partial^{2} \Phi}{\partial x^{2}} \\
& +k(\theta-v) \frac{\partial \Phi}{\partial v}+\frac{1}{2} \sigma^{2}\left(2 \theta v-\theta^{2}\right) \frac{\partial^{2} \Phi}{\partial v^{2}} \\
& +\frac{\partial^{2} \Phi}{\partial x \partial v}\left(\frac{3}{2} \theta^{1 / 2} v-\frac{1}{2} \theta^{3 / 2}\right) \sigma \rho+\lambda \Phi(x, v, \tau ; u) \Lambda(u)=0 .
\end{aligned}
$$

According to Duffie et al. [23], this PDE has an exponential-affine solution of the form

$$
\Phi(x, v, \tau ; u)=\exp (i u x+A(u, \tau)+B(u, \tau) v),
$$

with the boundary conditions

$$
A(u, 0)=B(u, 0)=0 .
$$

Substituting equation (15) into equation (14) yields

$$
\begin{aligned}
& -\left(\frac{\partial A}{\partial \tau} v+\frac{\partial B}{\partial \tau}\right)+\left(r-\lambda m-\frac{v}{2}\right) i u+\frac{1}{2} v(i u)^{2} \\
& +k(\theta-v) A+\frac{\sigma^{2}}{2}\left(2 \theta v-\theta^{2}\right) A^{2} \\
& +\left(\frac{3}{2} \theta^{1 / 2} v-\frac{1}{2} \theta^{3 / 2}\right) \sigma \rho i u A+\lambda \Lambda(u)=0 .
\end{aligned}
$$

By matching coefficients, we can derive the following two ordinary differential equations (ODEs): 


$$
\begin{aligned}
& \frac{\partial A}{\partial \tau}=\sigma^{2} \theta A^{2}+\left(\frac{3}{2} \theta^{1 / 2} \sigma \rho i u-k\right) A-\frac{1}{2}\left(i u+u^{2}\right) \\
& \frac{\partial B}{\partial \tau}=(r-\lambda m) i u+k \theta A-\frac{1}{2} \sigma^{2} \theta^{2} A^{2}-\frac{1}{2} \sigma \rho \theta^{3 / 2} i u A+\lambda \Lambda(u) .
\end{aligned}
$$

Since the ODE (18) is a Riccati equation, its general solution is

$$
A(u, \tau)=\alpha_{0} \frac{1-e^{-\alpha \tau}}{-\beta_{2}+\beta_{1} e^{-\alpha \tau}}
$$

where

$$
\begin{aligned}
& \alpha_{0}=-\frac{1}{2}\left(i u+u^{2}\right), \\
& \alpha_{1}=\frac{3}{2} \theta^{1 / 2} \sigma \rho i u-k, \\
& \alpha_{2}=\sigma^{2} \theta, \\
& \beta_{1}=\frac{\alpha_{1}+\alpha}{2}, \\
& \beta_{2}=\frac{\alpha_{1}-\alpha}{2}, \\
& \alpha=\sqrt{\alpha_{1}^{2}-4 \alpha_{0} \alpha_{2} .}
\end{aligned}
$$

Multiplying $\theta / 2$ on both sides of equation (18), and substituting it into equation (19), we can obtain:

$$
\begin{aligned}
\frac{\partial B}{\partial \tau}= & (r-\lambda m) i u+\left(\frac{1}{2} \theta k+\frac{1}{4} \sigma \rho i u \theta^{3 / 2}\right) A \\
& -\frac{1}{4} \theta\left(i u+u^{2}\right)-\frac{\theta}{2} \frac{\partial A}{\partial \tau}+\lambda \Lambda(u) .
\end{aligned}
$$

Integrating on both sides of the above equation, we have the following result:

$$
\begin{aligned}
B(\tau, u)= & -\frac{1}{2} \theta A(\tau, u)-\frac{\alpha_{3}}{\alpha_{2}}\left[\beta_{1} \tau+\ln \left(\frac{-\beta_{2}+\beta_{1} e^{-\alpha \tau}}{\alpha}\right)\right] \\
& +((r-\lambda m) i u+\lambda \Lambda(u)) \tau-\frac{1}{4} \theta\left(i u+u^{2}\right) \tau
\end{aligned}
$$

where

$$
\alpha_{3}=\frac{1}{2} \theta k+\frac{1}{4} \sigma \rho i u \theta^{3 / 2} .
$$

This finished the proof of Theorem 1.

Remark 1. Once that the analytic expression of the characteristic function has been obtained, the cumulant of $\ln S_{T}$ can be computed, which will be used in the truncation of the computational domain of option pricing. Following Fang and Oosterlee [18], the $n$-th cumulant of $\ln S_{T}$ is given by

$$
c_{n}=\left.\frac{1}{i^{n}} \frac{\partial^{n}(\ln \Phi(u))}{\partial u^{n}}\right|_{u=0} .
$$

3.2. Valuation of Discretely Monitored Barrier Options. It is well known that according to whether the underlying asset price needs to pass or to avoid a certain barrier level to receive a payoff and whether it has the barrier above or below the initial underlying asset price, barrier options can be classified into four cases, i.e., up-and-out option, up-and-in option, down-and-out option, down-and-in option. Without loss of generality, we take knock-out options as an example to illustrate the derivation of pricing formulas of barrier option in this section. The payoff functions are shown in Table 1 for different discretely monitored knock-out barrier options with strike $\mathrm{K}$, barrier level $\mathrm{H}$, and maturity $\mathrm{T}$.

Let $\quad x:=\ln \left(S_{t_{m-1}} / K\right) \quad$ and $\quad y:=\ln \left(S_{t_{m}} / K\right)$, $m=M, M-1, \ldots, 2$. Then the prices of discretely knock up-and-out and down-and-out barrier option, i.e., $v_{\mathrm{OU}}$ and $v_{\mathrm{OD}}$, can be expressed as the following recursive formulas:

$$
\begin{aligned}
& v_{\mathrm{OU}}\left(x, t_{m-1}\right)= \begin{cases}c\left(x, t_{m-1}\right), & x<h, \\
e^{-r\left(T-t_{m-1}\right)} \mathrm{Rb}, & x \geq h,\end{cases} \\
& v_{\mathrm{OD}}\left(x, t_{m-1}\right)= \begin{cases}e^{-r\left(T-t_{m-1}\right)} \mathrm{Rb}, & x \leq h, \\
c\left(x, t_{m-1}\right), & x>h,\end{cases}
\end{aligned}
$$

where

$$
c\left(x, t_{m-1}\right)=e^{-r \Delta t} \int_{\mathbb{R}} v\left(y, t_{m}\right) f(y \mid x) \mathrm{d} y,
$$

with

$$
v\left(x, t_{0}\right)=e^{-r \Delta t} \int_{\mathbb{R}} v\left(y, t_{1}\right) f(y \mid x) \mathrm{d} y,
$$

where $h=\ln H / K, K$ is the strike price, $T$ is the maturity, $M$ is the monitoring date and $H$ is the barrier level, $\mathrm{Rb}$ is a rebate, $r$ denotes the risk-free interest rate; $v(x, t)$ and $c(x, t)$ are the option value and continuation value at time t, respectively; $f(y \mid x)$ is the probability density of $\mathrm{y}$ given $\mathrm{x}$ under the risk-neutral measure $\mathbb{Q}$.

Usually, the probability density function $f(y \mid x)$ is unknown. Fortunately, Fang and Oosterlee [18] proposed an approximation of the probability density function with a truncated region $[a, b]$ as follows:

$$
f(y \mid x) \approx \frac{2}{b-a} \sum_{k=0}^{N-1^{\prime}} \operatorname{Re}\left\{\Phi\left(\frac{k \pi}{b-a}\right) e^{i k \pi(x-a / b-a)}\right\} \cos \left(k \pi \frac{y-a}{b-a}\right),
$$

where $\sum^{\prime}$ means the summation whose first term is multiplied by $1 / 2, \mathfrak{R}\{\cdot\}$ denotes taking the real part of a complex number. And $\Phi(u)$ is the conditional characteristic function of probability density function $f(y \mid x)$. 
TABLE 1: Payoff functions for different types of discretely monitored knock-out barrier options.

\begin{tabular}{lc}
\hline Option type & Payoff \\
\hline Up-and-out call & {$\left[\left(S_{T}-K\right)^{+}-\mathrm{Rb}\right] 1_{\left\{S_{t_{i}}<H\right\}}+\mathrm{Rb}$} \\
Up-and-out put & {$\left[\left(K-S_{T}\right)^{+}-\mathrm{Rb}\right] 1_{\left\{S_{t_{i}}<H\right\}}+\mathrm{Rb}$} \\
Down-and-out call & {$\left[\left(S_{T}-K\right)^{+}-\mathrm{Rb}\right] 1_{\left\{S_{t_{i}}>H\right\}}+\mathrm{Rb}$} \\
Down-and-out put & {$\left[\left(K-S_{T}\right)^{+}-\mathrm{Rb}\right] 1_{\left\{S_{t_{i}}>H\right\}}+\mathrm{Rb}$} \\
\hline
\end{tabular}

$t_{i}$ is the observation date.

Replacing the $f(y \mid x)$ in equation (26) with its approximation equation (29) and interchanging summation and integration, the approximation of the continuation value $c\left(x ; t_{m-1}\right)$ can be obtained:

$\widehat{c}\left(x, t_{m-1}\right)=e^{-r \Delta t} \frac{2}{b-a} \sum_{k=0}^{N-1} \mathfrak{R}^{\prime}\left\{\Phi\left(\frac{k \pi}{b-a}\right) e^{i k \pi(x-a / b-a)}\right\} V_{k}\left(t_{m}\right)$, where

$$
V_{k}\left(t_{m}\right)=\frac{2}{b-a} \int_{a}^{b} v\left(y, t_{m}\right) \cos \left(k \pi \frac{y-a}{b-a}\right) \mathrm{d} y .
$$

We know that barrier options are equivalent to European options at time $t \in\left[t_{0}, t_{1}\right]$. So the price of the discrete barrier option at initial time $t_{0}$ can be approximated as

$$
\widehat{v}\left(x, t_{0}\right)=e^{-r \Delta t} \sum_{k=0}^{N-1} \mathfrak{R}^{\prime}\left\{\Phi\left(\frac{k \pi}{b-a}\right) e^{i k \pi(x-a / b-a)}\right\} V_{k}\left(t_{1}\right) .
$$

Now the key point is to obtain the coefficient $V_{k}\left(t_{m}\right), m=M, M-1, \ldots, 1$. For the convenience of later description, we denote:

$$
\begin{aligned}
\chi_{k}\left(x_{1}, x_{2}\right) & =\int_{x_{1}}^{x_{2}} e^{x} \cos \left(k \pi \frac{x-a}{b-a}\right) d x \\
& =\frac{1}{1+(k \pi b-a)^{2}}\left[\cos \left(k \pi \frac{x_{2}-a}{b-a}\right) e^{x_{2}}-\cos \left(k \pi \frac{x_{1}-a}{b-a}\right) e^{x_{1}}+\frac{k \pi}{b-a} \sin \left(k \pi \frac{x_{2}-a}{b-a}\right) e^{x_{2}}-\frac{k \pi}{b-a} \sin \left(k \pi \frac{x_{1}-a}{b-a}\right) e^{x_{1}}\right], \\
\psi_{k}\left(x_{1}, x_{2}\right) & =\int_{x_{1}}^{x_{2}} \cos \left(k \pi \frac{x-a}{b-a}\right) \mathrm{d} x \\
& = \begin{cases}\frac{b-a}{k \pi}\left[\sin \left(k \pi \frac{x_{2}-a}{b-a}\right)-\sin \left(k \pi \frac{x_{1}-a}{b-a}\right)\right], & k \neq 0, \\
x_{2}-x_{1}, & k=0 .\end{cases}
\end{aligned}
$$

When $m=M$, if $h<0$, we have the following results:

$$
V_{k}\left(t_{M}\right)= \begin{cases}\frac{2}{b-a} \operatorname{Rb} \psi_{k}(h, b), & \text { for up - and - out call, } \\ \frac{2}{b-a}\left[\operatorname{Rb} \psi_{k}(a, h)+K \chi_{k}(0, b)-K \psi_{k}(0, b)\right], & \text { for down - and - out call, } \\ \frac{2}{b-a}\left[K \psi_{k}(a, h)-K \chi_{k}(a, h)+\mathrm{Rb} \psi_{k}(h, b)\right], & \text { for up - and - out put, } \\ \frac{2}{b-a}\left[\operatorname{Rb} \psi_{k}(a, h)+K \psi_{k}(h, 0)-K \chi_{k}(h, 0)\right], & \text { for down - and - out put. }\end{cases}
$$

If $h \geq 0$, then we can obtain the following result: 


$$
V_{k}\left(t_{M}\right)= \begin{cases}\frac{2}{b-a}\left[K \chi_{k}(0, h)-K \psi_{k}(0, h)+\mathrm{Rb} \psi_{k}(h, b)\right], & \text { for up - and - out call, } \\ \frac{2}{b-a}\left[\operatorname{Rb} \psi_{k}(a, h)+K \chi_{k}(h, b)-K \psi_{k}(h, b)\right], & \text { for down - and - out call, } \\ \frac{2}{b-a}\left[K \psi_{k}(a, 0)-K \chi_{k}(a, 0)+\mathrm{Rb} \psi_{k}(h, b)\right], & \text { for up - and - out put } \\ \frac{2}{b-a} \operatorname{Rb} \psi_{k}(a, h), & \text { for down - and - out put. }\end{cases}
$$

For $m=M-1, M-2, \ldots, 1$, we have the following theorem.
Theorem 2. For the up-and-out option and down-and-out option, the recurrent coefficients $V_{k}\left(t_{m}\right)$ can be approximately expressed as follows:

$$
\widehat{V}_{k}\left(t_{m}\right)= \begin{cases}\widehat{C}_{k}\left(a, h, t_{m}\right)+e^{-r\left(T-t_{m}\right)} \mathrm{Rb} \frac{2}{b-a} \psi_{k}(h, t b), & \text { up - and - out, } \\ e^{-r\left(T-t_{m}\right)} \mathrm{Rb} \frac{2}{b-a} \psi_{k}(a, h)+\widehat{C}_{k}\left(h, b, t_{m}\right), & \text { down - and - out }\end{cases}
$$

where $\widehat{C}_{k}\left(x_{1}, x_{2}, t_{m}\right)$ can be approximately calculated by:

$$
\widehat{C}_{k}\left(x_{1}, x_{2}, t_{m}\right)=e^{-r \Delta t} \operatorname{Re}\left\{\sum_{j=0}^{N-1} \Phi\left(\frac{j \pi}{b-a}\right) \widehat{V}_{j}\left(t_{m+1}\right) M_{k, j}\left(x_{1}, x_{2}\right)\right\} \text {, }
$$

with

$$
M_{k j}\left(x_{1}, x_{2}\right)=\frac{2}{b-a} \int_{x_{1}}^{x_{2}} e^{i j \pi(x-a / b-a)} \cos \left(k \pi \frac{x-a}{b-a}\right) \mathrm{d} x .
$$

Proof. According to the payoff of discrete barrier options at time $T$, we can rewrite $v(y, T)$ as follows:

$$
v(y, T)=\left[K \alpha\left(e^{y}-1\right)^{+}-\mathrm{Rb}\right] 1_{\left\{y_{t_{i}}<h\right\}}+\mathrm{Rb},
$$

where $\alpha=1$ for call option and $\alpha=-1$ for put option. Then we can obtain the above-mentioned results by the following computations.

For $m=M-1, M-2, \ldots, 1$, we have

$$
\begin{array}{r}
V_{k}\left(t_{m}\right)=\frac{2}{b-a} \int_{a}^{b} v\left(y, t_{m}\right) \cos \left(k \pi \frac{y-a}{b-a}\right) \mathrm{d} y \\
=\frac{2}{b-a} \int_{a}^{h} v\left(y, t_{m}\right) \cos \left(k \pi \frac{y-a}{b-a}\right) \mathrm{d} y+\frac{2}{b-a} \int_{h}^{b} v\left(y, t_{m}\right) \cos \left(k \pi \frac{y-a}{b-a}\right) \mathrm{d} y \\
=\frac{2}{b-a} \int_{a}^{h} c\left(y, t_{m}\right) \cos \left(k \pi \frac{y-a}{b-a}\right) \mathrm{d} y+\frac{2}{b-a} \int_{h}^{b} e^{-r\left(T-t_{m}\right)} \mathrm{Rb} \cos \left(k \pi \frac{y-a}{b-a}\right) \mathrm{d} y \\
=C_{k}\left(a, h, t_{m}\right)+e^{-r\left(T-t_{m}\right)} \mathrm{Rb} \frac{2}{b-a} \psi_{k}(h, b) .
\end{array}
$$

On the other hand, $c\left(y, t_{m}\right)$ can be approximated by $\widehat{c}\left(x, t_{m}\right)$, where

$$
\widehat{c}\left(x, t_{m}\right)=e^{-r \Delta t} \sum_{k=0}^{N-1^{\prime}} \operatorname{Re}\left\{\Phi\left(\frac{k \pi}{b-a}\right) e^{i k \pi(x-a / b-a)}\right\} V_{k}\left(t_{m+1}\right) .
$$


TABle 2: Parameter values for the numerical experiments.

\begin{tabular}{|c|c|c|c|c|c|c|c|c|c|c|c|c|c|c|}
\hline Parameter & $r$ & $\lambda$ & $k$ & $\theta$ & $\sigma$ & $p$ & $\eta_{1}$ & $T$ & $S_{0}$ & $\rho$ & $v_{0}$ & $H$ & $\mathrm{Rb}$ & $\eta_{2}$ \\
\hline Value & 0.05 & 3 & 5 & 0.2 & 0.7 & 0.3 & 10 & 1 & 100 & -0.5 & 0.2 & 120 & 5 & 5 \\
\hline
\end{tabular}

TABle 3: Comparisons of the CPU time and accuracy for Fouriercosine method(FC method) and the Monte Carlo simulation (MC Simulation) for pricing discrete up-and-out call barrier options.

\begin{tabular}{ccccc}
\hline M & $K$ & FC method & MC simulation & Abs R.E. \\
\hline \multirow{4}{*}{4} & 90 & 3.6231 & 3.6681 & 1.23 \\
& 100 & 2.8640 & 2.9086 & 1.53 \\
& 110 & 2.4709 & 2.5083 & 1.49 \\
& cpu time (sec) & 1.142 & 110.952 & \\
\hline \multirow{4}{*}{12} & 90 & 3.5703 & 3.6003 & 0.83 \\
& 100 & 3.0630 & 3.0909 & 0.90 \\
& 110 & 2.8221 & 2.8489 & 0.94 \\
& cpu time (sec) & 1.2721 & 144.136 & \\
\multirow{4}{*}{26} & 90 & 3.5709 & 3.6128 & 1.16 \\
& 100 & 3.1584 & 3.1882 & 0.93 \\
& 110 & 2.9740 & 2.9965 & 0.75 \\
& cpu time (sec) & 1.429 & 136.546 & \\
52 & 90 & 3.5815 & 3.6216 & 1.10 \\
& 100 & 3.2239 & 3.2511 & 0.83 \\
& 110 & 3.0713 & 3.0892 & 0.58 \\
& cpu time (sec) & 1.492 & 197.555 & \\
\hline \multirow{4}{*}{252} & 90 & 3.6075 & 3.6373 & 0.82 \\
& 100 & 3.3168 & 3.3343 & 0.52 \\
& 110 & 3.2021 & 3.2109 & 0.27 \\
& cpu time(sec) & 1.013 & 324.363 & \\
\hline
\end{tabular}

TABle 4: Comparisons of the CPU time and accuracy for Fouriercosine method (FC method) and the Monte Carlo simulation(MC Simulation) for pricing discrete up-and-out put barrier options.

\begin{tabular}{ccccc}
\hline M & $K$ & FC method & MC simulation & Abs R.E. (\%) \\
\hline \multirow{4}{*}{4} & 90 & 15.3051 & 15.2912 & 0.09 \\
& 100 & 19.2898 & 19.2349 & 0.29 \\
& 110 & 23.6405 & 23.5468 & 0.04 \\
& cpu time (sec) & 1.219 & 237.383 & \\
\hline \multirow{4}{*}{12} & 90 & 14.3355 & 14.2995 & 0.25 \\
& 100 & 17.7990 & 17.7157 & 0.47 \\
& 110 & 21.5291 & 21.4019 & 0.59 \\
& cpu time (sec) & 1.066 & 289.999 & \\
\multirow{4}{*}{26} & 90 & 13.8651 & 13.8281 & 0.27 \\
& 100 & 17.0935 & 17.0050 & 0.52 \\
& 110 & 20.5499 & 20.4138 & 0.67 \\
& cpu time (sec) & 1.099 & 136.546 & \\
52 & 90 & 13.5387 & 13.5097 & 0.21 \\
& 100 & 16.6115 & 16.5350 & 0.46 \\
& 110 & 19.8893 & 19.7695 & 0.61 \\
& cpu time (sec) & 1.383 & 155.311 & \\
\multirow{4}{*}{252} & 90 & 13.0696 & 13.0470 & 0.17 \\
& 100 & 15.9276 & 15.8641 & 0.40 \\
& 110 & 18.9614 & 18.8627 & 0.52 \\
& cpu time (sec) & 1.170 & 113.495 &
\end{tabular}

Substituting the above equation into $C_{k}\left(a, h, t_{m}\right)$, we can obtain $\widehat{C}_{k}\left(a, h, t_{m}\right)$ as follows: which is the approximate value of $C_{k}\left(a, h, t_{m}\right)$ :

$$
\begin{aligned}
\widehat{C}_{k}\left(a, h, t_{m}\right) & =\frac{2}{b-a} \int_{a}^{h} \widehat{c}\left(y, t_{m}\right) \cos \left(k \pi \frac{y-a}{b-a}\right) \mathrm{d} y \\
& =e^{-r \Delta t} \Re\left\{\sum_{j=0}^{N-1} \Phi^{\prime}\left(\frac{j \pi}{b-a}\right) V_{j}\left(t_{m+1}\right) \mathscr{M}_{k, j}(a, h)\right\},
\end{aligned}
$$

where

$$
\mathscr{M}_{k, j}(a, h):=\frac{2}{b-a} \int_{a}^{h} e^{i j \pi(x-a / b-a)} \cos \left(k \pi \frac{x-a}{b-a}\right) \mathrm{d} x .
$$

Hence, $V_{k}\left(t_{m}\right)$ can be recovered from $V_{k}\left(t_{m+1}\right)$. Finally, we substitute the approximation $\widehat{V}_{k}\left(t_{1}\right)$ into equation (28) to calculate the option price $\widehat{v}\left(x, t_{0}\right)$.

\section{Numerical Analysis}

4.1. Comparison of the Approximate Solutions against Monte Carlo Simulations. In this section, some numerical examples are performed to show the performance of the Fouriercosine method for pricing discrete barrier options against its alternative competitor, the Monte Carlo simulation, as a benchmark. We perform numerical examples on the discretely monitored up-and-out call and up-and-out put barrier options. Following Fang and Oosterlee [19], the integration interval $[a, b]$ is chosen as follows:

$$
[a, b]=\left[c_{1}+a_{0}-L \sqrt{c_{2}+\sqrt{c_{4}}}, c_{1}+a_{0}+L \sqrt{c_{2}+\sqrt{c_{4}}}\right] \text {, }
$$

with $a_{0}=\ln S_{0}, L=10$ and $c_{n}$ is the $n$-th cumulant of $\ln S_{T}$.

For Monte Carlo method, we use 100,000 numbers of simulations, 200 numbers of time steps. Let $N=2^{10}$ for Fourier-cosine method. The parameter values are listed in Table 2 for all our numerical examples. The computer used in the experiments equips an Intel Core i3 CPU with a 2.53 $\mathrm{GHZ}$ processor. And all of our numerical examples were performed using Matlab 2016a.

Our numerical results show that the Fourier-cosine method is fast and accurate for pricing discrete up-and-out barrier options. The absolute relative error (Abs R.E.) and CPU time information, comparing the Fourier-cosine method and the Monte Carlo simulation, are presented in Tables 3 and 4 for pricing discrete up-and-out call and put barrier options. Tables 3 and 4 also compares relative errors between the Fourier-cosine method and the Monte Carlo simulation for pricing discrete up-and-out call and put barrier options and they show that the Fourier-cosine method is significantly faster than Monte Carlo simulation. Furthermore, Tables 3 and 4 compare the pricing accuracy between the two methods across a range of strike prices, and 


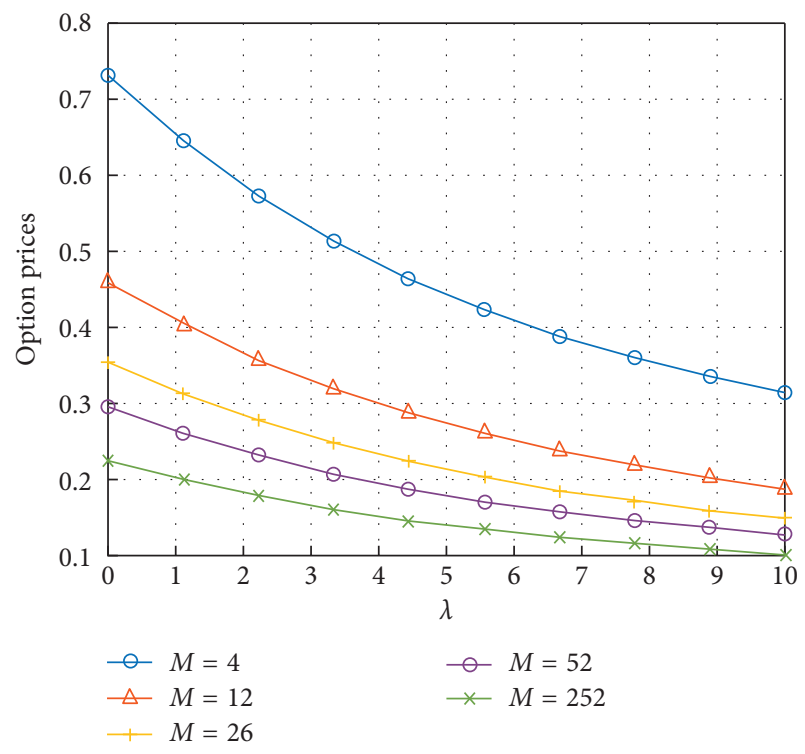

FIgURE 1: Prices of discrete up-and-out call barrier options with respect to the parameters of $\lambda$.

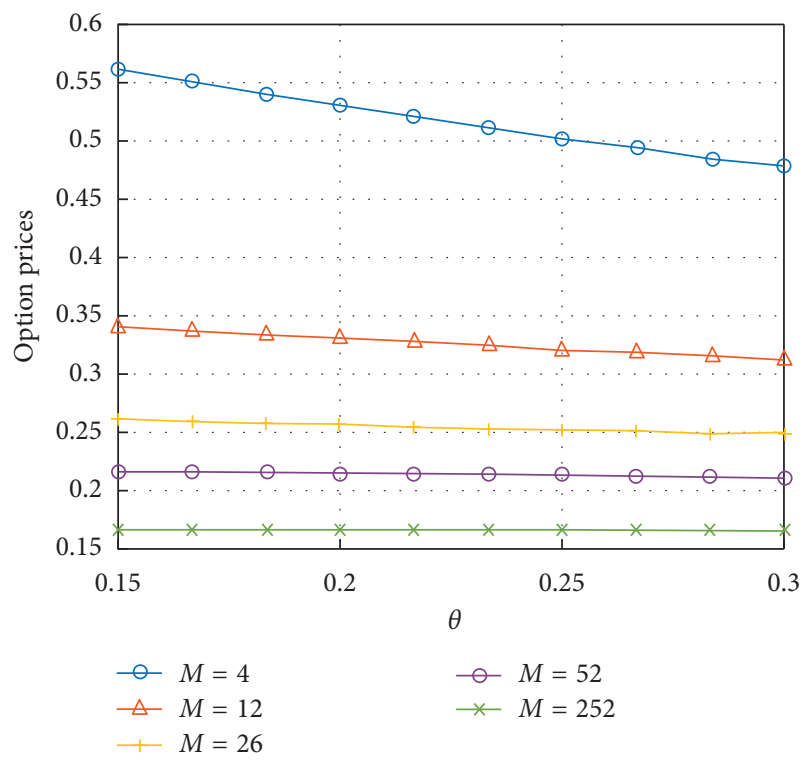

Figure 2: Prices of discrete up-and-out call barrier options with respect to $\theta$.

the relative percentage of price differences of Fourier-cosine method are all less than $1.6 \%$. Therefore, numerical results show that the Fourier-cosine method is accurate and effective.

4.2. Sensitivity Analysis of the Model Parameters. In this section, we will evaluate the impact of parameters on pricing discretely monitored up-and-out call barrier options. We mainly study the effects of the following parameters on the price of discretely monitored up-and-out call barrier options: (i) the jump intensity $\lambda$, (ii) the long-run mean level $\theta$,

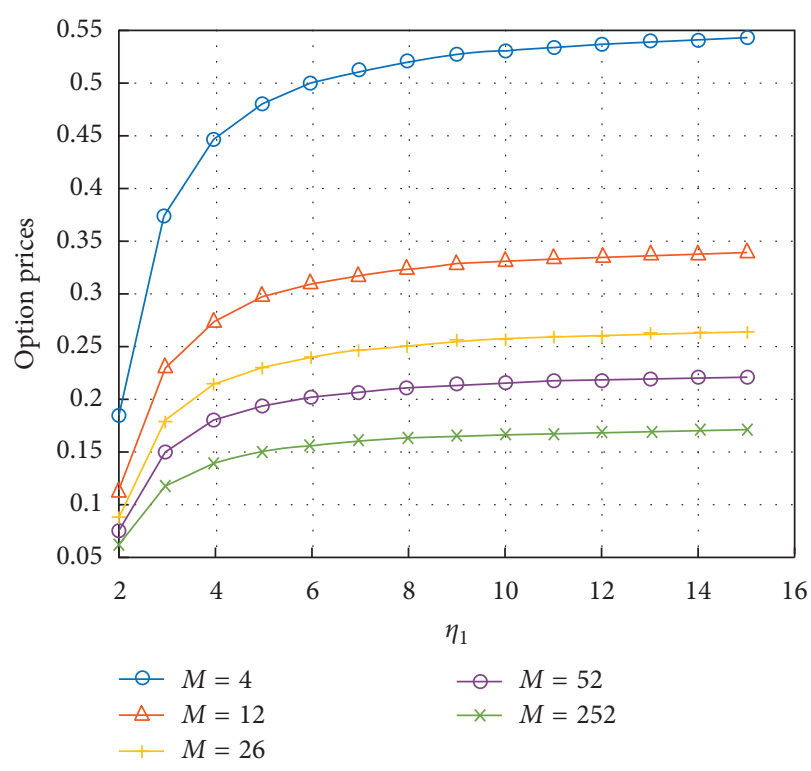

FIgURE 3: Prices of discrete up-and-out call barrier options with respect to the parameters of $\eta_{1}$.

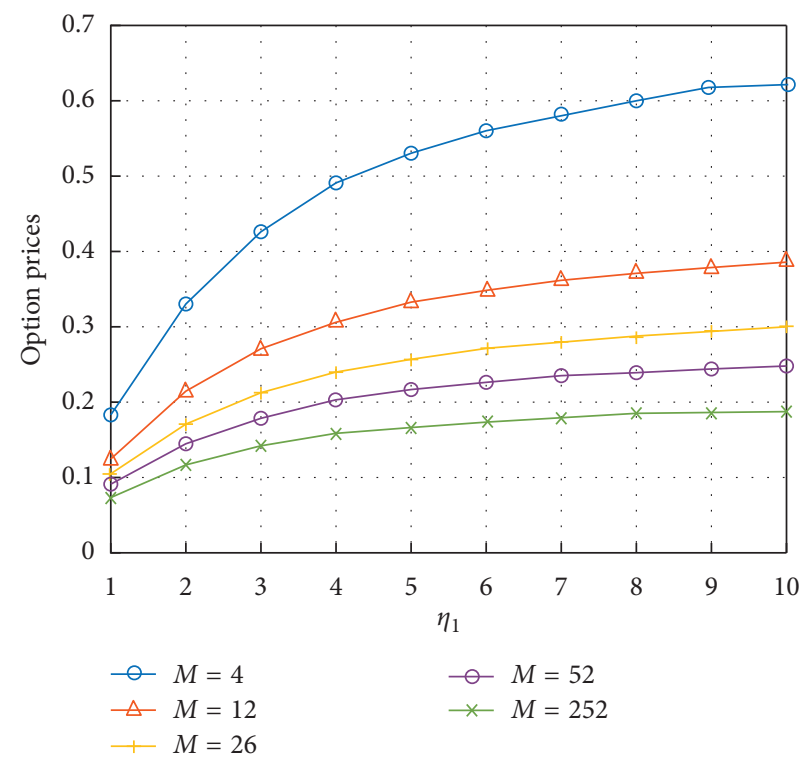

FIgURE 4: Prices of discrete up-and-out call barrier options with respect to the parameters of $\eta_{2}$.

(iii) the reciprocal of the mean for the positive jumps $\eta_{1}$, and (iv) the reciprocal of the mean for the negative jumps $\eta_{2}$.

Figures 1 and 2 show the variation trend of the price of the discrete knock up-out call barrier option with respect to the jump intensity $\lambda$ and the long-run mean level $\theta$, respectively. As shown in Figure 1, a higher jump intensity $\lambda$ induces a lower price of discrete knock up-out call barrier option. The explanation for this phenomenon is that it makes the underlying asset price process more variable as the jump intensity $\lambda$ increases. So it makes the underlying asset price more likely to cross the barrier level $\mathrm{H}$ at the monitoring dates. Therefore, the price of discrete up-and- 
out call barrier option is a decreasing function of the jump intensity $\lambda$. The implication of Figure 2 can be expressed in the similar way.

Figures 3 and 4 illustrate the effects of the reciprocal of the mean for the positive jumps $\eta_{1}$, and the negative jumps $\eta_{2}$ on the price of discrete knock up-out call barrier option. We can see that the price of discrete knock up-out call barrier option increases with respect to the parameters $\eta_{1}$ and $\eta_{2}$, respectively. The reason is that the probability density function of the underlying log-asset price becomes more leptokurtosis and heavy tails when the parameters $\eta_{1}$ and $\eta_{2}$ increase.

\section{Conclusion}

This paper proposed an efficient method for the valuating the discrete barrier options when the underlying asset price process is governed by the GARCH volatility and double exponential jump. We utilized Taylor expansion method to derive an analytical approximation of the characteristic function for the underlying log-asset price. Based on Fourier-cosine method, a quasianalytical approximate formula of the price of the discrete barrier is obtained. The Fourier-cosine method is fast and efficient for pricing discrete barrier options compared with Monte Carlo simulation. Finally, the sensitivities of some parameters in the model are provided to explain our results.

\section{Data Availability}

The data used to support the findings of this study are available from the corresponding author upon request.

\section{Conflicts of Interest}

The authors declare no conflicts of interest.

\section{Acknowledgments}

This research was financially supported by the Fundamental Research Funds for the Central Universities JBK2002003, and the authors gratefully acknowledge this support.

\section{References}

[1] F. Black and M. Scholes, "The pricing of options and corporate liabilities," Journal of Political Economy, vol. 81, no. 3, pp. 637-654, 1973.

[2] R. C. Merton, "Theory of rational option pricing," The Bell Journal of Economics and Management Science, vol. 4, no. 1, pp. 141-183, 1973.

[3] D. Rich, "The mathematical foundations of barrier optionpricing theory," Advances in Futures and Options Research, vol. 7, pp. 267-311, 1994.

[4] M. Rubinstein and E. Reiner, "Breaking down the barriers," Risk, vol. 4, pp. 28-35, 1991.

[5] S. G. Kou and H. Wang, "Option pricing under a double exponential jump diffusion model," Management Science, vol. 50, no. 9, pp. 1178-1192, 2004.

[6] L. Feng and V. Linetsky, "Pricing discretely monitored barrier options and defaultable bonds in lvy process models: a fast hilbert transform approach," Mathematical Finance, vol. 18, no. 3, pp. 337-384, 2008.

[7] G. Fusai, G. Germano, D. Marazzina, and S. identity, "Spitzer identity, Wiener-Hopf factorization and pricing of discretely monitored exotic options," European Journal of Operational Research, vol. 251, no. 1, pp. 124-134, 2016.

[8] G. Lian, S.-P. Zhu, R. J. Elliott, and Z. Cui, "Semi-analytical valuation for discrete barrier options under time-dependent Lévy processes," Journal of Banking \& Finance, vol. 75, pp. 167-183, 2017.

[9] A. Sobhani and M. Milev, "A numerical method for pricing discrete double barrier option by legendre multiwavelet," Journal of Computational and Applied Mathematics, vol. 328, pp. 355-364, 2018.

[10] N. Thakoor, D. Y. Tangman, M. Bhuruth et al., "A spectral approach to pricing of arbitrage-free SABR discrete barrier options," Computational Economics, vol. 54, no. 3, pp. 1085-1111, 2019.

[11] Z. Li, W.-G. Zhang, Y.-J. Liu, and Y. Zhang, "Pricing discrete barrier options under jump-diffusion model with liquidity risk," International Review of Economics \& Finance, vol. 59, pp. 347-368, 2019.

[12] E. M. Stein and J. C. Stein, "Stock price distributions with stochastic volatility: an analytic approach," Review of Financial Studies, vol. 4, no. 4, pp. 727-752, 1991.

[13] S. L. Heston, "A closed-form solution for options with stochastic volatility with applications to bond and currency options," Review of Financial Studies, vol. 6, no. 2, pp. 327-343, 1993.

[14] D. B. Nelson, "ARCH models as diffusion approximations," Journal of Econometrics, vol. 45, no. 1-2, pp. 7-38, 1990.

[15] P. Christoffersen, K. Jacobs, and K. Mimouni, "Volatility dynamics for the S\&P500: evidence from realized volatility, daily returns, and option prices," Review of Financial Studies, vol. 23, no. 8, pp. 3141-3189, 2010.

[16] K. Chourdakis and G. Dotsis, "Maximum likelihood estimation of non-affine volatility processes," Journal of Empirical Finance, vol. 18, no. 3, pp. 533-545, 2011.

[17] Z. Huang, C. Tong, T. Wang et al., "Which volatility model for option valuation in China? empirical evidence from SSE 50 ETF options," Applied Economics, vol. 52, no. 17, pp. 1866-1880, 2020.

[18] F. Fang and C. W. Oosterlee, "A novel pricing method for European options based on fourier-cosine series expansions," SIAM Journal on Scientific Computing, vol. 31, no. 2, pp. 826-848, 2008.

[19] F. Fang and C. W. Oosterlee, "Pricing early-exercise and discrete barrier options by fourier-cosine series expansions," Numerische Mathematik, vol. 114, no. 1, pp. 27-62, 2009.

[20] F. Fang and C. W. Oosterlee, "A fourier-based valuation method for bermudan and barrier options under heston's model," SIAM Journal on Financial Mathematics, vol. 2, no. 1, pp. 439-463, 2011.

[21] S. Zhang and J. Geng, "Fourier-cosine method for pricing forward starting options with stochastic volatility and jumps," Communications in Statistics-Theory and Methods, vol. 46, no. 20, pp. 9995-10004, 2017.

[22] R. Caldana and G. Fusai, "A general closed-form spread option pricing formula," Journal of Banking \& Finance, vol. 37, no. 12, pp. 4893-4906, 2013.

[23] D. Duffie, J. Pan, K. Singleton et al., "Transform analysis and asset pricing for affine jump-diffusions," Econometrica, vol. 68, no. 6, pp. 1343-1376, 2000. 